\title{
DIFFUSION LIMIT OF THE LORENTZ MODEL: ASYMPTOTIC PRESERVING SCHEMES *
}

\author{
Christophe Buet $^{1}$, Stéphane Cordier ${ }^{2}$, Brigitte Lucquin-Desreux ${ }^{3}$ And Simona \\ MANCINI $^{3}$
}

\begin{abstract}
This paper deals with the diffusion limit of a kinetic equation where the collisions are modeled by a Lorentz type operator. The main aim is to construct a discrete scheme to approximate this equation which gives for any value of the Knudsen number, and in particular at the diffusive limit, the right discrete diffusion equation with the same value of the diffusion coefficient as in the continuous case. We are also naturally interested with a discretization which can be used with few velocity discretization points, in order to reduce the cost of computation.
\end{abstract}

Mathematics Subject Classification. 82C70, 35B40, 65N06.

Received: December 12, 2001. Revised: March 1, 2002.

\section{INTRODUCTION}

In this article, we study the numerical schemes for a kinetic equation in the diffusive regime:

$$
\varepsilon \partial_{t} f+\cos \theta \partial_{x} f=\frac{1}{\varepsilon} \mathcal{L}(f)
$$

The problem is one-dimensional in the space variable $x$ and bi-dimensional in the velocity variable $v=$ $(\cos \theta, \sin \theta)$. The unknown distribution function $f=f(x, \theta, t)$ is a function of position $x(x \in \mathbb{R}$ : in this article we shall not consider boundary conditions), of the velocity angle $\theta \in[-\pi, \pi]$ and of time $t>0$. The operator $\mathcal{L}(f)$ is a linear collision operator of Lorentz type. Lorentz operators appear for example when considering elastic collisions of heavy particles (e.g. ions) against light ones (e.g. electrons); it is the first order term of the inter-species collision operator representing the collisions of the heavy particles on the light ones, when doing an asymptotic expansion in terms of the small mass ratio (see $[8,35])$. This operator does not depend on the energy variable, i.e. on the modulus of the velocity. It is defined in the Boltzmann case by (for simplicity,

\footnotetext{
Keywords and phrases. Hilbert expansion, diffusion limit.

* The authors acknowledge support from the TMR project "Asymptotic methods in kinetic theory" (TMR number: ERB FMRX CT97 0157), run by the European Community.

1 CEA/DAM Ile de France, BP 12, 91680 Bruyères-Le-Châtel, France. e-mail: Christophe.Buet@cea.fr

2 Laboratoire MAPMO, UMR 6628, Université d'Orléans, 45067 Orléans, France. e-mail: Stephane.Cordier@univ-orleans.fr

3 Laboratoire d'Analyse Numérique, UMR 7598, Université Pierre et Marie Curie, BP 187, 75252 Paris Cedex 05, France. e-mail: lucquin@ann.jussieu.fr \& smancini@ann.jussieu.fr
} 
we drop the dependence with respect to $x$ and $t$, since the collision operator only acts on $\theta$ ):

$$
\mathcal{L}(f)(\theta)=\int_{S^{1}} K\left(\theta^{\prime}-\theta\right)\left[f\left(\theta^{\prime}\right)-f(\theta)\right] \mathrm{d} \theta^{\prime}
$$

and in the Fokker-Planck case by:

$$
\mathcal{L}(f)(\theta)=\partial_{\theta \theta}^{2} f .
$$

We recall that the Boltzmann-Lorentz operator (1.2) converges (up to multiplication by the second order moment of the scattering cross-section $K$ ) to the Fokker-Planck-Lorentz one (1.3) when the cross-section concentrates, i.e. when the scattering angle during a collision, $\theta^{\prime}-\theta$, is very small. This is the so-called "grazing collision limit" [3,6,36], which is also valid in the non-linear case $[7,9]$.

It is well known in literature, that for $\varepsilon \ll 1$ the solution of (1.1) converges to the solution of a diffusion problem, with respect to the space variable. The diffusion coefficient of this last equation may be computed by means of a Hilbert expansion method (see Sect. 2). Our goal is to derive a numerical scheme that is relevant for any value of $\varepsilon$. For simplicity, we will consider the Fokker-Planck-Lorentz collision operator defined by (1.3). Nevertheless, we remark that our results can be extended to the Boltzmann-Lorentz collision operator defined by $(1.2)$.

This paper is inspired by a series of articles written by Jin and Levermore about diffusive limit of the isotropic Boltzmann-Lorentz operator, where the cross-section is constant with respect to the velocity variable. This fact does not allow to pass to the grazing collision limit, excluding thus the Fokker-Planck case. The authors consider discrete ordinate methods in the velocity variable, or equivalently in the cosine of the angle (the distribution function being isotropic in the others directions). More precisely, they determine the quadrature points in the integral with respect to velocity such that, in the diffusion limit, one recovers a heat equation with the correct diffusion coefficient. A first paper, see [22], is devoted to the discretization in velocities, the distribution function depending continuously of the space and time variables. The authors construct quadrature sets corresponding to a small number of discrete velocities such that the diffusion coefficient and also the boundary conditions are compatible with the diffusive limit. In a second paper, see [23], they investigate the fully discrete case (both on the velocity and the space variables), the problem being stationary. This can be seen as the problem for one time step iteration using an implicit scheme. In another paper with Golse, see [13], they study the convergence of theses schemes.

Our aim is to extend this analysis to other elastic collision operators with an arbitrary cross-section (i.e. not necessarily constant) and for example in the case of the grazing collision limit, i.e. for the Fokker-Planck Lorentz operator (1.3).

There is a huge literature on related topics taking different names. We claim that the following keywords are used to qualify very close problematic although the goal and the methods used are different.

\section{- Diffusive $(a=1)$ or hydrodynamic $(a=0)$ limit}

$$
\varepsilon^{a} \partial_{t} f+\cos \theta \partial_{x} f=\frac{1}{\varepsilon} \mathcal{L}(f) .
$$

The aim is to derive numerical schemes which can be used either in rarefied (where $\varepsilon$ is of order 1 ) or in dense regions $(\varepsilon \ll 1)$. Depending on the collision operator $\mathcal{L}$, one expects the solution to converge towards an equilibrium state (typically a Maxwellian distribution) and that the conserved quantities are solution of the Euler equations (for $a=0$ ) or Navier-Stokes equations (for $a=1$ ), see [26, 29, 43, 44].

A lot of works have been devoted to the coupling of rarefied and hydro-dynamical domains. For this purpose, numerical schemes compatible with the fluid limit, called Asymptotic preserving schemes, are an alternative to the matching of boundary conditions, these last being sometimes hard to design. In fact, these schemes can work uniformly with respect to the relaxation parameter (see [27] and its introduction for more details). 


\section{- Stiff source term for hyperbolic systems}

A widely studied topic deals with hyperbolic systems of conservation laws (e.g. Euler equations) with stiff source terms (see $[4,5,28])$ due, for example, to the modeling of rapid chemical reactions. These systems read:

$$
\partial_{t} U+\partial_{x} F(U)=\frac{1}{\varepsilon} S(f)
$$

and are related to the so called relaxation methods (see $[24,28,40,41]$ ) that consists in replacing a nonlinear problem of the above form by a relaxed linear system of the following form:

$$
\partial_{t} U+\partial_{x} V=0, \quad \partial_{t} V+a \partial_{x} U=\frac{-1}{\varepsilon}(V-F(U))
$$

where $\varepsilon$ is called relaxation rate and $a$ is an arbitrary velocity (with $-\sqrt{a}<F^{\prime}(U)<\sqrt{a}$ ).

We remark that there is another area in the hyperbolic systems field that is related to such asymptotic namely the kinetic schemes: in this direction, one replaces the hyperbolic system of interest (e.g. the Euler equation) by a kinetic formulation in the hydro-dynamical limit (e.g. a B.G.K type equation), see $[34,42]$. Then, one derives a scheme on the kinetic formulation and takes its hydro-dynamical limit. These methods have stability, accuracy and efficiency advantages. Some results on convergence are also available, see [2].

Let us also mention another recently way to design Asymptotic Preserving Schemes, the Well Balanced schemes, see $[14,16,17,33]$, designed to capture stationary fluxes for hyperbolic systems with source terms. In [14-16] such a scheme is used for Goldstein-Taylor type models (two characteristic speeds). A Chapman-Enskog expansion shows that the asymptotic limit of the scheme is the good one for GoldsteinTaylor type models, but on systems that have more than two characteristic speeds the asymptotic seems hard to generalize.

There is a large number of applications based on transport equations having a diffusive asymptotic. Let us mention, for instance, neutron transport, radiative transfer in the "optically thick limit" (see $[1,31,32,38,39]$ and the references therein) and semiconductor modeling (see $[25,29,37]$ ). However, many of the previous works deal with the so-called telegrapher equation, or equivalently Goldstein-Taylor equation which is a kinetic equation where the distribution function is localized on two opposite velocities (see $[4,20,21,26,27,40]$ ). On the other hand, in this paper, we shall not separate the particle density function with respect to the sign of the velocity, usually called parity method or even-odd decomposition. We refer more precisely to the introduction of Section 4.4 for a detailed explanation of this fact and a comparison with previous works. Let us finally mention that the space discretization scheme we propose could be also apply to the Boltzmann-Lorentz operator with an arbitrary cross-section.

This work is divided as follows. In Section 2, we briefly recall the Hilbert expansion method at the continuous level in order to derive the diffusion model. Then, we consider successively the discretization with respect to the velocity angle $\theta$, to the space variable $x$, and finally to the time $t$.

In Section 3, following Jin-Levermore (see [22,23], for what regards the isotropic Boltzmann-Lorentz collision operator), we consider the problem discretized only with respect to the velocity variable. We look for a choice of velocity discretization points such that the diffusion coefficient tends towards the value obtained in the continuous case. Our goal is also to use a small number (denoted by $N_{\theta}$ ) of discretization points with respect to velocity angle, in order to avoid too much expensive computations. We prove that if we consider a uniform grid, we obtain the right diffusion coefficient only in the limit $N_{\theta} \rightarrow \infty$ (with an accuracy of order 2). In order to use a kinetic description with a small number of discretization points and to preserve the right asymptotic when $\varepsilon \rightarrow 0$, it is thus necessary to consider a non-uniform (but symmetric) grid. In particular, we treat the case of a grid with 4 or 8 discretization points.

In Section 4, we consider the space discretization (the velocity discretization is assumed to be known). More precisely, we study the discretization of the transport term $v \partial_{x} f$. The boundary layer problem is not taken 
into account, and will be studied in futures works. The first approach consists of discretizing this term by means of an upwind scheme, but this method leads to a parasite solvability condition, i.e. we get an infinite diffusion coefficient. On the contrary, the centered scheme converges to a discretization of the Laplacian (with respect to $x$ ), but the discretization acts on a double mesh, the even points of the mesh being decoupled from the odd points, yielding to some spurious modes (which gives numerical oscillations in the computations). We then consider a $\theta$-scheme $(\varepsilon$ part for the upwind scheme and $(1-\varepsilon)$ for the centered one): the first part effectively re-couples the discretization points, but it introduces an error of order $\Delta x$ in the value of the diffusion coefficient. Finally, we take into account the modified Jin-Levermore scheme which is obtained following the strategy proposed by Jin and Levermore (see [24,28]). It consists in writing a finite volume type scheme and in computing the fluxes at the interfaces using the leading order term (or equivalently, the steady state equation) in the upwind scheme for the half mesh. We can also give an interpretation of this scheme as a finite discontinuous elements $P^{1}$ scheme (see [45]).

Section 5 deals with the time discretization. First, we remark that the usual methods of splitting transport and collisions are not suitable in the limit $\varepsilon \rightarrow 0$. In fact, both the collision and the transport parts yield to projecting onto the constant states (with respect to velocity or position), and in only two time iterations one may get a constant function. Moreover, to avoid the CFL condition, which would lead to a very small time step when $\varepsilon \rightarrow 0$, we must use implicit schemes. Numerical results are given in Section 6. Some final results and comments are then presented as a conclusion in Section 7.

\section{The Diffusion Limit AT The CONTINUOUS LEVEL}

Let us first recall the derivation of the diffusion equation from the kinetic one by means of the Hilbert expansion method. As explained in the introduction, we consider the following kinetic equation of unknown $f=f(x, \theta, t)$ in one space variable $x \in \mathbb{R}$, the angular velocity being $\theta \in(0,2 \pi)$ with periodic condition on $\theta$, at the diffusion scale $\left(t / \varepsilon^{2}, x / \varepsilon\right)$ :

$$
\varepsilon \partial_{t} f+\cos \theta \partial_{x} f=\frac{1}{\varepsilon} \frac{\partial^{2} f}{\partial \theta^{2}},
$$

where the collision term is the Fokker-Planck-Lorentz operator and $\varepsilon \ll 1$ denotes the Knudsen number. We now use a classical Hilbert method by expanding $f$ in terms of $\varepsilon$ :

$$
f=f^{0}+\varepsilon f^{1}+\varepsilon^{2} f^{2}+\ldots,
$$

and identifying terms of equal powers in (2.1). We successively obtain:

$$
\begin{aligned}
\frac{\partial^{2} f^{0}}{\partial \theta^{2}} & =0, \\
\cos \theta \partial_{x} f^{0} & =\frac{\partial^{2} f^{1}}{\partial \theta^{2}}, \\
\partial_{t} f^{0}+\cos \theta \partial_{x} f^{1} & =\frac{\partial^{2} f^{2}}{\partial \theta^{2}} .
\end{aligned}
$$

From the first equation (2.2) and periodicity, we deduce that $f^{0}$ is independent of the velocity angle $\theta$. The solvability condition for the second equation writes:

$$
\int_{0}^{2 \pi} \cos \theta \partial_{x} f^{0} \mathrm{~d} \theta=0
$$


and this condition is actually satisfied, because $f^{0}$ does not depend on $\theta$ and $\int_{0}^{2 \pi} \cos \theta \mathrm{d} \theta=0$. Moreover, we have (up to the addition of a function which only depends on the space variable):

$$
f^{1}=-\cos \theta \partial_{x} f^{0}
$$

Finally, the solvability condition for equation (2.4) writes: $2 \pi \partial_{t} f^{0}+\int_{0}^{2 \pi} \cos \theta \partial_{x} f^{1} \mathrm{~d} \theta=0$, which, on account of (2.6) and the fact that $\int_{0}^{2 \pi} \cos ^{2} \theta \mathrm{d} \theta=\pi$ gives:

$$
\partial_{t} f^{0}-\frac{1}{2} \partial_{x x}^{2} f^{0}=0
$$

This is the limit diffusion equation we obtain at the continuous level with the diffusion coefficient $\nu=1 / 2$.

\section{The Velocity DiscRetization}

In this part, we consider the discretization with respect to the velocity variable, the time and space variables remaining continuous. This is the so-called discrete ordinate method. We shall perform the same analysis as in the continuous case (see Sect. 2). Our goal is to choose a small number of discretization points such that we obtain a discrete version of the diffusion equation (2.7) with still the right diffusion coefficient, i.e. $\nu=1 / 2$. Let us first consider a uniform grid.

\subsection{Uniform grid}

Let us define the uniform discretization of $S^{1}$ as the angle $\theta_{j}=j \Delta \theta$ with $\Delta \theta=2 \pi / N_{\theta}$ and $j=0 \ldots N_{\theta}-1$, where the indices $j$ are denoted modulo $N_{\theta}$ : i.e. $j=N_{\theta}$ corresponds to the same velocity as $j=0$. The discretized version of (2.1) is a finite system of transport equations coupled by the collision term:

$$
\varepsilon \partial_{t} f_{j}+\cos \theta_{j} \partial_{x} f_{j}=\frac{1}{\varepsilon}(L f)_{j}
$$

where $f_{j}(x, t)=f\left(x, \theta_{j}, t\right)$ is the value of the discretized distribution function $f$ at time $t$, position $x$ and velocity $\left(\cos \theta_{j}, \sin \theta_{j}\right)$. The operator $L$ (with entries $\left.L_{i j}\right)$ in the right hand side of equation (3.1) stands for the discretized version of the Laplacian operator with respect to the variable $\theta$. Using standard finite difference techniques and taking into account periodic boundary conditions, we obtain:

$$
L_{i j}=\frac{1}{\Delta \theta^{2}} \begin{cases}-2 & \text { if } i=j \\ 1 & \text { if }|i-j| \equiv 1[N]\end{cases}
$$

Let us recall some useful spectral properties of the matrix $L$ :

- The kernel of the matrix $L$ is generated by the vector $\mathbf{1}=(1,1,1, \ldots, 1)$.

- The vector $\left(\cos \theta_{j}\right)_{j=0 \ldots N_{\theta-1}}$ is an eigenvector of matrix $L$; the associated eigenvalue is $-\lambda$ where $\lambda$ is the positive number given by:

$$
\lambda=2 \frac{1-\cos (\Delta \theta)}{(\Delta \theta)^{2}}
$$

Performing the Hilbert expansion on the discretized problem (3.1), i.e.: $f_{j}=f_{j}^{0}+\varepsilon f_{j}^{1}+\varepsilon^{2} f_{j}^{2}+\ldots$ and identifying terms of equal powers of $\varepsilon$, we get:

$$
\begin{aligned}
\left(L f^{0}\right)_{j} & =0, \\
\cos \theta_{j} \partial_{x} f^{0} & =\left(L f^{1}\right)_{j}, \\
\partial_{t} f^{0}+\cos \theta_{j} \partial_{x} f^{1} & =\left(L f^{2}\right)_{j} .
\end{aligned}
$$


At the order $\varepsilon^{-1}$, we obtain that $f_{j}^{0}$ does not depend on $\theta_{j}$, since the kernel of $L$ consists of constant vectors; we simply set: $f_{j}^{0}=f^{0}$. Now, the solvability condition for equation (3.4) reads: $\sum_{j=0}^{N_{\theta}-1} \cos \theta_{j} \partial_{x} f^{0}=0$, which, because $f^{0}$ is independent of $\theta_{j}$, leads to:

$$
\sum_{j=0}^{N_{\theta}-1} \cos \theta_{j}=0 .
$$

We can interpret relation (3.6) as a symmetry condition. This condition is satisfied by our choice of discretization points (with $N_{\theta}$ even), but it does not hold if we translate the discretization points into $\theta_{0}+2 \pi j / N_{\theta}$ with $\theta_{0} \neq 0$ for example. We look for a solution $f^{1}$ of (3.4) orthogonal to the kernel of $L\left(\operatorname{Ker}(L)^{\perp}=\{f:\langle f, \mathbf{1}\rangle=0\}\right)$, i.e. such that:

$$
\sum_{j=0}^{N_{\theta}-1} f_{j}^{1}=0 .
$$

Then, as $f^{0}$ is independent of $\theta_{j}$ and $\left(\theta_{j}\right)$ satisfies (3.6), the unique solution of (3.4) reads:

$$
f_{j}^{1}=-\frac{\cos \theta_{j}}{\lambda} \partial_{x} f^{0}
$$

Finally, considering (3.8), equation (3.5) becomes: $\partial_{t} f^{0}-\frac{\cos ^{2} \theta_{j}}{\lambda} \partial_{x x}^{2} f^{0}=\left(L f^{2}\right)_{j}$. The solvability condition for this equation $\left(\sum_{j}\left(L f^{2}\right)_{j}=0\right)$ writes:

$$
\partial_{t} f^{0}-\frac{1}{2 \lambda} \partial_{x x} f^{0}=0
$$

because we have: $\left(1 / N_{\theta}\right) \sum_{j} \cos ^{2} \theta_{j}=1 / 2$. We then get a diffusion equation for $f^{0}$, but with a diffusion coefficient $\nu=\frac{1}{2 \lambda}$ : in fact, this coefficient is never equal to the expected value $1 / 2$ (since $\lambda \neq 1$, see (3.2)), but it converges towards it when $N_{\theta}$ tends to $\infty$ (with an accuracy of order $(\Delta \theta)^{2}$ ). Nevertheless, if we want to deal with small number of points in $\theta$, for lowering the computational cost, we have to investigate non-uniform grids. When using a large number of points, one can use an uniform discretization.

\subsection{Non-uniform grid}

First, let us briefly recall the discretization of the second derivative with respect to the angle using nonuniform grids, in order to introduce the notations. Given a discretization of $S^{1}, \theta_{j}$ for $j=0 \ldots N_{\theta}-1$, let us denote $\Delta_{j}^{+}=\left(\theta_{j+1}-\theta_{j}\right)$ and $\Delta_{j}^{-}=\left(\theta_{j}-\theta_{j-1}\right)$. Applying a Taylor expansion with respect to the point $\theta_{j}$ we obtain:

$$
\begin{aligned}
& f\left(\theta_{j}+\Delta_{j}^{+}\right)=f_{j}+\Delta_{j}^{+} f_{j}^{\prime}+\frac{\Delta_{j}^{+2}}{2} f_{j}^{\prime \prime}+o\left(\Delta_{j}^{+2}\right), \\
& f\left(\theta_{j}-\Delta_{j}^{-}\right)=f_{j}-\Delta_{j}^{-} f_{j}^{\prime}+\frac{\Delta_{j}^{-2}}{2} f_{j}^{\prime \prime}+o\left(\Delta_{j}^{-2}\right) .
\end{aligned}
$$

By combining these Taylor expansions, we get the following approximation of the second derivative at $\theta_{j}$ :

$$
f_{j}^{\prime \prime} \simeq 2 \frac{f\left(\theta_{j}+\Delta_{j}^{+}\right)}{\Delta_{j}^{+}\left(\Delta_{j}^{+}+\Delta_{j}^{-}\right)}+2 \frac{f\left(\theta_{j}-\Delta_{j}^{-}\right)}{\Delta_{j}^{-}\left(\Delta_{j}^{+}+\Delta_{j}^{-}\right)}-2 \frac{f_{j}}{\Delta_{j}^{+} \Delta_{j}^{-}} .
$$


We shall discretize the Laplace operator using this formula when $N_{\theta}=4$ or 8 . We remark that in the general case the vector with components $\cos \theta_{j}$ is no more an eigenvector of $L$, as it is easily seen replacing $f_{j}$ by $\cos \theta_{j}$ in $(3.10)$.

Let us perform the same discretized Hilbert expansion as presented in the uniform grid case (Sect. 3.1). At first order, $f_{j}^{0}=f^{0}$ is independent on $\theta_{j}$. Let us define $Y \in \mathbb{R}^{N_{\theta}}$ as the unique solution of

$$
(L Y)_{j}=\cos \theta_{j}, \quad \text { for all } j \in\left\{0, \ldots, N_{\theta}-1\right\}
$$

such that $\sum_{j=0}^{N_{\theta}-1} Y_{j}=0$. Then, the unique solution $f^{1}$ of equation (3.4) satisfying (3.7) is given by:

$$
f^{1}=Y \partial_{x} f^{0}
$$

Equation (3.9) now reads: $\partial_{t} f^{0}+\cos \theta_{j} Y_{j} \partial_{x x}^{2} f^{0}=\left(L f^{2}\right)_{j}$, and the solvability condition for this equation of unknown $f^{2}$ still gives a diffusion equation for $f^{0}$ where the diffusion coefficient $\nu$ is now given by:

$$
\nu=-\frac{1}{N_{\theta}} \sum_{j=0}^{N_{\theta}-1} Y_{j} \cos \theta_{j}=-\frac{1}{N_{\theta}} L Y \cdot Y .
$$

We shall now show that for $N_{\theta}=4$ or $N_{\theta}=8$, one can construct symmetric sets of discretization points that give the good diffusion coefficient. This can be related to the analysis presented in $[22,23]$ for the Boltzmann-Lorentz operator.

\section{4 points of discretization}

Let us first consider the case $N_{\theta}=4$, i.e. 4 points of discretization in velocity: $\theta_{0}, \theta_{1}, \theta_{2}, \theta_{3}$. We still consider a symmetric discretization, i.e. such that (3.6) holds.

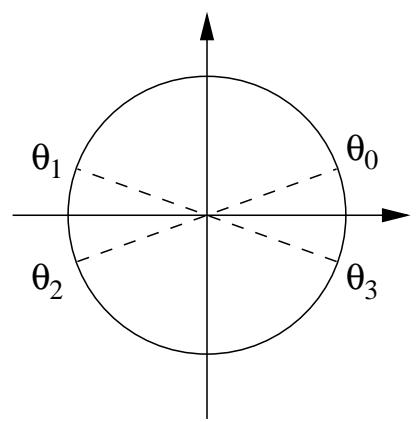

FIGURE 1. 4 discretization points in velocity.

More precisely, we choose $\theta_{0}, \theta_{1}=\pi-\theta_{0}, \theta_{2}=\pi+\theta_{0}, \theta_{3}=2 \pi-\theta_{0}$ i.e. $\cos \theta_{0}=-\cos \theta_{1}=-\cos \theta_{2}=\cos \theta_{3}$ (see Fig. 1). The goal is to determine $\theta_{0}$ in such a way that the associated diffusion coefficient $\nu$ is exactly equal to $1 / 2$. 
The discrete Laplacian matrix corresponding to this nonuniform grid reads:

$$
L=2\left(\begin{array}{cccc}
\frac{-1}{\Delta_{0} \Delta_{3}} & \frac{1}{\Delta_{0}\left(\Delta_{0}+\Delta_{3}\right)} & 0 & \frac{1}{\Delta_{3}\left(\Delta_{0}+\Delta_{3}\right)} \\
\frac{1}{\Delta_{0}\left(\Delta_{0}+\Delta_{1}\right)} & \frac{-1}{\Delta_{0} \Delta_{1}} & \frac{1}{\Delta_{1}\left(\Delta_{0}+\Delta_{1}\right)} & 0 \\
0 & \frac{1}{\Delta_{1}\left(\Delta_{1}+\Delta_{2}\right)} & \frac{-1}{\Delta_{1} \Delta_{2}} & \frac{1}{\Delta_{2}\left(\Delta_{1}+\Delta_{2}\right)} \\
\frac{1}{\Delta_{3}\left(\Delta_{2}+\Delta_{3}\right)} & 0 & \frac{1}{\Delta_{2}\left(\Delta_{2}+\Delta_{3}\right)} & \frac{-1}{\Delta_{2} \Delta_{3}}
\end{array}\right)
$$

where we denote by $\Delta_{0}=\theta_{1}-\theta_{0}, \Delta_{1}=\theta_{2}-\theta_{1}, \Delta_{2}=\theta_{3}-\theta_{2}, \Delta_{3}=\theta_{0}-\theta_{3}$ the discretization steps.

Proposition 3.1. Given $N_{\theta}=4$, there exists an unique value of $\theta_{0}$ such that we have the right diffusion coefficient, i.e. $\nu=1 / 2$.

Proof. We have to find $Y=\left(Y_{0}, Y_{1}, Y_{2}, Y_{3}\right) \in \mathbb{R}^{4}$ such that: $L Y=(\cos \theta)$ with $\sum_{j=0}^{3} Y_{j}=0$, where the notation $(\cos \theta)$ denotes the vector with components $(\alpha,-\alpha,-\alpha, \alpha)$, with $\alpha=\cos \theta_{0}$. After some easy computations, we find

$$
Y=-B(\cos \theta), \quad \text { with } B=\pi(\pi-2 \theta) / 4,
$$

and the diffusion coefficient is given by $\nu\left(\theta_{0}\right)=-\frac{1}{4} Y \cdot(\cos \theta)=B \alpha^{2}$. In order to find the right diffusion coefficient, we now have to look for a value $\theta_{0} \in[0, \pi / 2]$ such that $\nu\left(\theta_{0}\right)=1 / 2$, where

$$
\nu(\theta)=\frac{\pi}{4}(\pi-2 \theta) \cos ^{2} \theta .
$$

We have $\nu(0)=\pi^{2} / 4>1 / 2, \nu(\pi / 2)=0<1 / 2$ and $\nu^{\prime}\left(\theta_{0}\right)<0$. Thus, there exists a unique $\left.\theta_{0} \in\right] 0, \pi / 2[$ such that $\nu\left(\theta_{0}\right)=1 / 2$.

Surprisingly, for the non uniform (but symmetric) discrete point, the vector with components $\cos \theta_{j}$ is an eigenvector, associated with the eigenvalue $-B^{-1}$, for the matrix $L$. An approximated value of $\theta_{0}$, namely $\theta_{0}=0.8462$ has been computed using Maple ${ }^{\circledR}$. This value differs from $\pi / 4$, which is the case of uniform grid, but it is close to it.

\section{8 points of discretization}

We now consider the case $N_{\theta}=8$, i.e. 8 points of discretization in velocity. Let us denote by $\theta_{j}$ with $j=0, \ldots, 7$ a general 8-points discretization of $S^{1}$.

We choose a symmetric discretization, i.e. such that condition (3.6) holds, as follows: $\theta_{0}$ and $\theta_{1}$ belonging to $[0, \pi / 2]$ (the others angles being induced by this choice (see Fig. 2)). We now have two angles $\left(\theta_{0}\right.$ and $\theta_{1}$ ) that we shall determine in such a way that first the diffusion coefficient is the right one, and secondly that $Y$ is an eigenvector (this second condition is arbitrary; we add it in order to determine the two angles in a unique way and by analogy with previous cases). The discretization step, which we recall is not fixed, is given by:

$$
\begin{gathered}
\Delta_{0}=\theta_{1}-\theta_{0}, \quad \Delta_{1}=\theta_{2}-\theta_{1}=\pi-2 \theta_{2}, \quad \Delta_{2}=\theta_{3}-\theta_{2}=\theta_{1}-\theta_{0}, \\
\Delta_{3}=\theta_{4}-\theta_{3}=2 \theta_{0}, \quad \Delta_{4}=\theta_{5}-\theta_{4}=\theta_{1}-\theta_{0}, \quad \Delta_{5}=\theta_{6}-\theta_{5}=\pi-2 \theta_{1}, \\
\Delta_{6}=\theta_{7}-\theta_{6}=\theta_{1}-\theta_{0}, \quad \Delta_{7}=\theta_{0}-\theta_{7}=2 \theta_{0} .
\end{gathered}
$$




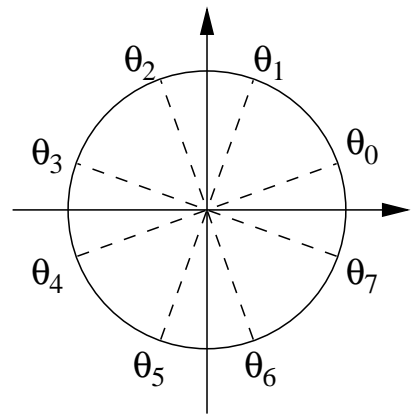

FiguRE 2. 8 discretization points in velocity.

We also impose that $\theta_{1}=m \theta_{0}$ for $0<\theta_{0}<\pi / 2$ and $1<m<\frac{\pi}{2 \theta_{0}}$. With the same method applied in the case of 4 velocities, we can derive the discretized version of the Laplacian $L$ :

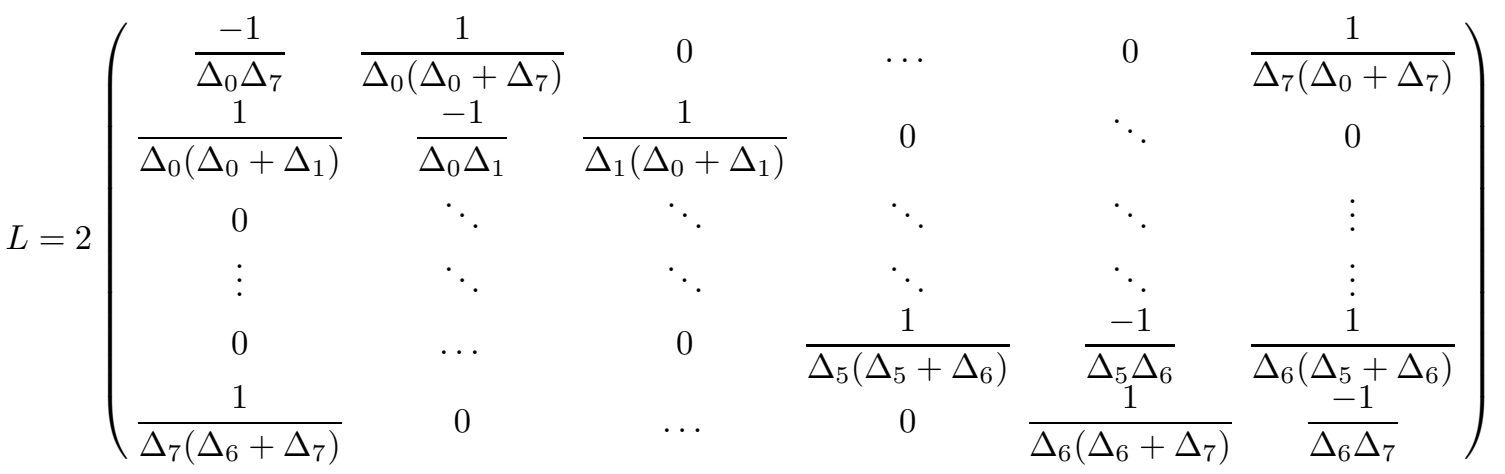

$$
\begin{aligned}
& =2\left(\begin{array}{cccccccc}
B & B_{1} & 0 & 0 & 0 & 0 & 0 & B_{2} \\
A_{1} & A & A_{2} & 0 & 0 & 0 & 0 & 0 \\
0 & A_{2} & A & A_{1} & 0 & 0 & 0 & 0 \\
0 & 0 & B_{1} & B & B_{2} & 0 & 0 & 0 \\
0 & 0 & 0 & B_{2} & B & B_{1} & 0 & 0 \\
0 & 0 & 0 & 0 & A_{1} & A & A_{2} & 0 \\
0 & 0 & 0 & 0 & 0 & A_{2} & A & A_{1} \\
B_{2} & 0 & 0 & 0 & 0 & 0 & B_{1} & B
\end{array}\right)
\end{aligned}
$$

where we have set

$$
\begin{array}{lll}
A=-1 / \alpha \gamma, & A_{1}=1 / \gamma(\gamma+\alpha), & A_{2}=1 / \alpha(\gamma+\alpha), \\
B=-1 / \beta \gamma, & B_{1}=1 / \gamma(\gamma+\beta), & B_{2}=1 / \beta(\gamma+\beta),
\end{array}
$$

with: $\alpha=\pi-2 m \theta_{0}, \beta=2 \theta_{0}$ and $\gamma=\theta_{0}(m-1)$. We still look for a vector $Y \in \mathbb{R}^{8}$ solution of $(3.11)$ and such that it is an eigenvector for the matrix $L$, i.e.:

$$
L Y=(\cos \theta)=-\lambda Y \text {. }
$$


We remark that now the vector $(\cos \theta)$ is given by $(\cos \theta)=(\bar{\alpha}, \bar{\beta},-\bar{\beta},-\bar{\alpha},-\bar{\alpha},-\bar{\beta}, \bar{\beta}, \bar{\alpha})$, where $\bar{\alpha}=\cos \theta_{0}$ and $\bar{\beta}=\cos \theta_{1}=\cos \left(m \theta_{0}\right)$. From system (3.13) we get the following system:

$$
\begin{aligned}
2 B_{1}(\bar{\beta}-\bar{\alpha}) & =-\lambda \bar{\alpha} \\
-2 A_{1}(-\bar{\alpha}+\bar{\beta})-4 \bar{\beta} A_{2} & =-\lambda \bar{\beta} .
\end{aligned}
$$

We recall that $\alpha, \beta$ and $\gamma$ depend on $\left(m, \theta_{0}\right)$ so that $A, A_{1}, A_{2}, B, B_{1}$ and $B_{2}$ also depend $\left(m, \theta_{0}\right)$. Thus, equation (3.14) yields to

$$
(\pi-2 m \theta)(\pi-m \theta-\theta)(\cos (m \theta)-\cos (\theta)) \cos (m \theta)=(m \theta+\theta) \cos (\theta)[(\pi-2 m \theta) \cos (\theta)-(\pi-2 \theta) \cos (m \theta)] .
$$

It remains to determine $\theta_{0}$ and $m$ such that the diffusion coefficient $\nu$ is equal to $(1 / 2)$ i.e. such that:

$$
\left.-2\left(\cos \left(m \theta_{0}\right)-\cos \theta_{0}\right)\right)=\left(m^{2}-1\right) \theta_{0}^{2}\left(\cos ^{2} \theta_{0}+\cos ^{2}\left(m \theta_{0}\right)\right) \cos \theta_{0}
$$

Solving $(3.15,3.16)$ using Maple, we get : $\theta_{0}=0.4318$ and $m=2.764$.

\section{The SPACE Discretization}

We use a finite difference discretization (see [11]) in order to approximate the transport problem (2.1). We recall that the aim is to derive a discrete model which at the limit $\varepsilon \rightarrow 0$ gives a good approximation of the diffusion equation (2.7). The velocity discretization is done using the finite difference scheme described in Section 3 (in particular, we shall set $N_{\theta}=4$ in the simulations), and we recall that $L$ is the discrete operator approximating the Laplacian $\frac{\partial^{2}}{\partial \theta^{2}}$.

We now describe different schemes for the convective part and study the asymptotic properties of the global space-velocity scheme. In all the sequel, we denote by $f_{i, j}$ an approximation of $f\left(x_{i}, \theta_{j},.\right)$, where $x_{i}=i \Delta x$ are the points of discretization in space variable (for simplicity we set $h=\Delta x$ ), and $v_{j}=\left(\cos \theta_{j}, \sin \left(\theta_{j}\right)\right.$ ), $j \in\left\{0, \ldots, N_{\theta}-1\right\}$ are those with respect to the velocity variable. We recall in particular that these points $\theta_{j}$ are symmetric in the sense that they satisfy the symmetry condition (3.6), which is also the discrete analogous of the continuous solvability condition (2.5). We also recall that these points are not necessarily equi-distributed, and in particular that for $N_{\theta}$ small $\left(N_{\theta}=4\right.$ for example ) we have shown that, in order to recover the good diffusion coefficient, these points are necessarily distributed in a non uniform way (we still refer to Sect. 3). Furthermore, denoting by $Y$ the vector in $\mathbb{R}^{N_{\theta}}$ such that $(L Y)_{j}=\cos \theta_{j}$, for all $j \in\left\{0, \ldots, N_{\theta}-1\right\}$ with $\sum Y_{j}=0$, the discrete diffusion coefficient is then given by (3.12) and at least in the simple cases $N_{\theta}=4$ or 8 there exists a unique choice of such discrete points $\theta_{j}$ such that $\nu$ is the good diffusion coefficient, i.e. such that:

$$
\nu=\frac{1}{N_{\theta}} \sum_{j} Y_{j} \cos \theta_{j}=\frac{1}{2}
$$

In what follows, the space-velocity discretized kinetic equation reads, for all $i, j$ :

$$
\varepsilon \partial_{t} f_{i, j}+\cos \theta_{j}(D f)_{i, j}=\frac{1}{\varepsilon}\left(L f_{i, .}\right)_{j},
$$

where $D$ denotes a finite difference operator approximating the first derivative with respect to the space variable. 


\subsection{The upwind scheme}

The more natural way to discretize the first derivative $\partial_{x} f$ is the upwind scheme $D^{u}$, which is defined by:

$$
D^{u} f_{i, j}= \begin{cases}\frac{f_{i, j}-f_{i-1, j}}{h}, & \text { if } \cos \theta_{j}>0, \\ \frac{f_{i+1, j}-f_{i, j}}{h}, & \text { if } \cos \theta_{j}<0 .\end{cases}
$$

We consider the discrete equation (4.1) with $D=D^{u}$ and use an Hilbert expansion, for all $i, j$

$$
f_{i, j}=f_{i, j}^{0}+\varepsilon f_{i, j}^{1}+\varepsilon^{2} f_{i, j}^{2}+\ldots
$$

Identifying terms of equal powers, we get, for all $i, j$ :

$$
\begin{aligned}
\left(L f_{i, .}^{0}\right)_{j} & =0, \\
\cos \theta_{j}\left(D^{u} f^{0}\right)_{i, j} & =\left(L f_{i, .}^{1}\right)_{j}, \\
\partial_{t} f_{i, j}^{0}+\cos \theta_{j}\left(D^{u} f^{1}\right)_{i, j} & =\left(L f_{i, .}^{2}\right)_{j} .
\end{aligned}
$$

Equation (4.3) shows that $f^{0}$ does not depend on $j$. Now, equation (4.4) is solvable only if the following solvability condition is fulfilled:

$$
\sum_{j=0}^{N_{\theta}-1} \cos \theta_{j}\left(D^{u} f^{0}\right)_{i, j}=0
$$

But, on account of condition (3.6), we deduce that this is equivalent to:

$$
f_{i+1}^{0}-2 f_{i}^{0}+f_{i-1}^{0}=0
$$

which is the discrete approximation of a stationary diffusion equation. This is not the good diffusion limit we expected, since it gives an infinite diffusion coefficient $\nu=\infty$. Hence, this scheme is not asymptotic preserving, since it gives a wrong diffusion equation at the limit $\varepsilon \rightarrow 0$.

\subsection{The centered scheme}

Let us now examine the centered scheme which we shall denote by $D^{c}$ :

$$
D^{c} f_{i, j}=\frac{f_{i+1, j}-f_{i-1, j}}{2 h} .
$$

The discrete problem is then (4.1) with $D=D^{c}$. Identifying again the terms of equal powers in the Hilbert expansion (4.2) we get, for all $i, j$ :

$$
\begin{aligned}
\left(L f_{i, .}^{0}\right)_{j} & =0, \\
\cos \theta_{j}\left(D^{c} f_{., j}^{0}\right)_{i} & =\left(L f_{i, .}^{1}\right)_{j}, \\
\partial_{t} f_{i, j}^{0}+\cos (\theta)_{j}\left(D^{c} f_{., j}^{1}\right)_{i} & =\left(L f_{i, .}^{2}\right)_{j} .
\end{aligned}
$$

Equation (4.6) shows that $f^{0}$ still does not depend on $j$. The solvability condition of equation (4.7) is then automatically satisfied, on account of the symmetry property (3.6). Moreover, we have:

$$
f_{i, j}^{1}=Y_{j}\left(D^{c} f^{0}\right)_{i},
$$


where $Y$ is given by (3.11). The solvability condition for equation (4.8) writes:

$$
N_{\theta} \partial_{t} f_{i}^{0}+\sum_{j=0}^{N_{\theta}-1} \cos \theta_{j}\left(D^{c} f_{., j}^{1}\right)_{i}=0
$$

which gives, on account of (4.9) and of the definition of $\nu$, the following discrete diffusion equation:

$$
\partial_{t} f_{i}^{0}-\nu\left(\Delta_{2 h} f^{0}\right)_{i}=0,
$$

where we have used the notation:

$$
\left(\Delta_{2 h} \phi\right)_{i}=\frac{\phi_{i+2}-2 \phi_{i}+\phi_{i-2}}{(2 h)^{2}} .
$$

In the same way, we shall denote by $\Delta_{h}$ the classical "three points scheme":

$$
\left(\Delta_{h} \phi\right)_{i}=\frac{\phi_{i+1}-2 \phi_{i}+\phi_{i-1}}{h^{2}} .
$$

Although the discrete equation (4.10) is a consistent approximation of the continuous diffusion equation (2.7), we remark that this scheme will generate numerical oscillations, due to spurious modes: the discrete points corresponding to an even index $i$ do not influence those corresponding to an odd one. We have actually observed this phenomena, by doing numerical experiments with an initial data of Dirac type (see Fig. 3). Since this scheme generates numerical oscillations, we drop it.

\subsection{The $\varepsilon$ scheme $D^{\varepsilon}$}

An interesting idea however is to combine this scheme to the upwind one (since the last scheme gives a diffusion operator of type $\Delta_{h}$ instead of $\Delta_{2 h}$ ), in order to avoid this phenomena. But we have seen that the upwind scheme does not give the right diffusion equation, on account of the solvability condition obtained when identifying constant terms. So a good compromise consist in studying the following convex combination:

$$
D^{\varepsilon}=(1-\varepsilon) D^{c}+\varepsilon D^{u} ;
$$

we shall denote it by " $\varepsilon$ - scheme".

The discrete problem is here (4.1) with $D=D^{\varepsilon}$ and the Hilbert expansion method gives, for all $i, j$ :

$$
\begin{aligned}
\left(L f_{i, .}^{0}\right)_{j} & =0, \\
\cos \theta_{j}\left(D^{c} f^{0}\right)_{i, j} & =\left(L f_{i, .}^{1}\right)_{j}, \\
\partial_{t} f_{i, j}^{0}+\cos \theta_{j}\left[\left(D^{c} f_{., j}^{1}\right)_{i}-\left(D^{c} f_{., j}^{0}\right)_{i}+\left(D^{u} f^{0}\right)_{i, j}\right] & =\left(L f_{i, .}^{2}\right)_{j} .
\end{aligned}
$$

From equation (4.11), we still have that $f^{0}$ independent of $j$, and the solvability condition for equation (4.12) is fulfilled, on account of (3.6). Moreover, as (4.12) coincide with (4.7), we have (4.9). The solvability condition for equation (4.13) is then:

$$
N_{\theta} \partial_{t} f_{i}^{0}+\sum_{j=0}^{N_{\theta}-1} \cos \theta_{j}\left(D^{c} f_{., j}^{1}\right)_{i}+\sum_{j=0}^{N_{\theta}-1} \cos \theta_{j}\left(D^{u} f^{0}\right)_{i, j}=0
$$


which gives, on account of expression (4.9), of the definition of $\nu$ and of the condition (3.6), the following discrete diffusion equation:

$$
\partial_{t} f_{i}^{0}-\nu\left(\Delta_{2 h} f^{0}\right)_{i}-\nu^{\prime}\left(\Delta_{h} f^{0}\right)_{i}=0
$$

where we have set:

$$
\nu^{\prime}=\frac{h}{2 N_{\theta}} \sum_{j=0}^{N_{\theta}-1}\left|\cos \theta_{j}\right| .
$$

This equation is still a consistent approximation of the diffusion equation (2.7) and it links the points with even index to those with an odd one. Thus, we can expect this scheme to suppress the oscillations given by the pure centered scheme. This has been effectively observed numerically, but for an initial data which support contains at least two grid points (in space variable). For a Dirac type initial data however, the oscillations still persist (see Fig. 4). This scheme thus seems to be a relatively good one, although the diffusion coefficient is not exactly the good one (up to an $0(h)$ error). We now examine a last scheme which seems to have all the expected properties.

\subsection{The modified Jin-Levermore scheme}

This new scheme is inspired by the scheme proposed by Jin and Levermore for the telegraph problem in [24] (which is based on the use of the steady states approximation method, see for example $[10,47], \ldots$ ). In this work, Jin and Levermore studied semi-discrete numerical schemes for hyperbolic systems with stiff relaxation terms that have a long time behavior governed by reduced systems of parabolic type, but the diffusion terms are in fact corrective terms of order $\varepsilon$. A similar problem, but under diffusive scaling, called the Goldstein-Taylor model (see $[12,46])$, has been numerically considered by [26]: here, both the convective terms and the relaxation terms are stiff (like for our problem), which gives additional difficulties. One of the mean ideas consists in writing the stiff terms as source terms, then to use a splitting in time algorithm, treating the relaxation terms by an implicit scheme and the new non-stiff convective terms by classical upwind schemes (or more accurate second order schemes with slope limiters). Special care has been taken to assure that the scheme possess the corrective diffusive limit. An extension of this work to more general source terms have been recently considered, in [26] for a bi-dimensional Boltzmann-Lorentz type operator, and for the linear Boltzmann equation in [25]. Both works are concerned with the actual diffusive regime and are both based on a splitting of the distribution into its even and odd part, giving then a system which is treated with similar discretization than the Goldstein-Taylor model previously described. A new difficulty arises in the Boltzmann case to solve the collision step implicitly: one needs in fact to invert an integral operator, which is not easy to do in an efficient way. A velocity discretization using Hermite polynomials is then performed.

It is in fact possible to adapt this method here to our problem, by first splitting $f$ into an even and an odd part and then using the discrete algorithm proposed in [25]. Moreover, let us point out that the implicit treatment of the collision part is here far simpler for our model than for the Boltzmann case considered in [25]. For the continuous in time scheme, we obtain the good diffusion limit with the right coefficient of diffusion. For the full discrete problem, we obtain the right diffusion coefficient, up to an $O(h)$ error, like for the $\varepsilon$ scheme proposed in Section 4.3.

It is one of the reasons why we decided to construct a new scheme, still based on Jin-Levermore's approach, but where it would be possible to directly discretize $f$ (without needing to split it into and even and an odd part) which would be far simpler. Moreover, the splitting method proposed in [22] seems interesting in so far the discretization with respect to the space variable is "unconnected" from the velocity one, but as the stiff convective terms appear in the right hand side of the relaxation part, it seems that both meshes are coupled, giving this scheme less attractive. Finally, we point out that the scheme proposed below can be also applied to the Boltzmann-Lorentz operator with an arbitrary cross-section. 
The idea for the construction of our new scheme is based on an evaluation of the fluxes at the interface between the two cells $\left[x_{i}-h / 2, x_{i}+h / 2\right]$ and $\left[x_{i+1}-h / 2, x_{i+1}+h / 2\right]$. The scheme writes $(4.1)$, with $D=D^{1 / 2}$ defined by:

$$
\left(D^{1 / 2} f\right)_{i, j}=\frac{f_{i+1 / 2, j}-f_{i-1 / 2, j}}{h}
$$

The idea for the evaluation of the interface values $f_{i+1 / 2, j}$ (or $f_{i-1 / 2, j}$ ) is first to write (up to an $O\left(h^{2}\right)$ error) the Taylor expansion of $f_{i+1 / 2, j}$ :

$$
\begin{aligned}
f_{i, j} & \simeq f_{i+1 / 2, j}-\frac{h}{2}\left(\partial_{x} f_{., j}\right)_{i+1 / 2}, \quad \text { if } \cos \theta_{j}>0, \\
f_{i+1, j} & \simeq f_{i+1 / 2, j}+\frac{h}{2}\left(\partial_{x} f_{., j}\right)_{i+1 / 2}, \quad \text { if } \cos \theta_{j}<0,
\end{aligned}
$$

which corresponds to a classical upwind scheme, according to the sign of the velocity. Now, in order to compute the space gradients, we consider the following continuous equation, with respect to the space variable only, i.e.

$$
\varepsilon \partial_{t} f+\cos \theta \partial_{x} f=\frac{1}{\varepsilon} L f
$$

in which we neglect the lowest order terms, with respect to $\varepsilon$, i.e. the $O(\varepsilon)$ term. This gives the following system (for simplicity, we suppose that, from now on, we never have $\cos \theta_{j}=0$ ):

$$
\begin{aligned}
f_{i, j} & =f_{i+1 / 2, j}-\frac{h}{2 \varepsilon \cos \theta_{j}}\left(L f_{i+1 / 2, .}\right)_{j}, \quad \text { if } \cos \theta_{j}>0, \\
f_{i+1, j} & =f_{i+1 / 2, j}+\frac{h}{2 \varepsilon \cos \theta_{j}}\left(L f_{i+1 / 2, .}\right)_{j}, \quad \text { if } \cos \theta_{j}<0 .
\end{aligned}
$$

In other words, the interface values satisfy:

$$
\left[\left(I d-\frac{h}{2 \varepsilon\left|\cos \theta_{j}\right|} L\right) f_{i+1 / 2, .}\right]_{j}=\left\{\begin{array}{cl}
f_{i, j}, & \text { if } \cos \theta_{j}>0 \\
f_{i+1, j}, & \text { if } \cos \theta_{j}<0
\end{array}\right.
$$

We now use the Hilbert method, expanding $f_{i, j}$ according to (4.2) and identifying terms of equal powers in the kinetic equation (4.1); we get, for all $i, j$ :

$$
\begin{aligned}
\left(L f_{i, .}^{0}\right)_{j} & =0, \\
\cos \theta_{j}\left(D^{1 / 2} f^{0}\right)_{i, j} & =\left(L f_{i, .}^{1}\right)_{j}, \\
\partial_{t} f_{i, j}^{0}+\cos \theta_{j}\left(D^{1 / 2} f^{1}\right)_{i, j} & =\left(L f_{i, .}^{2}\right)_{j} .
\end{aligned}
$$

The first equation shows, as usual, that $f_{i, j}^{0}$ does not depend on $j$. Now, in order to solve the other equations, we also need to expand $f_{i+1 / 2, j}$ in terms of $\varepsilon$. We set for all $i, j$

$$
f_{i+1 / 2, j}=f_{i+1 / 2, j}^{0}+\varepsilon f_{i+1 / 2, j}^{1}+\varepsilon^{2} f_{i+1 / 2, j}^{2}+\ldots
$$


and identify terms of equal powers in equations $(4.14,4.15)$, this gives:

$$
\begin{aligned}
& \left(L f_{i+1 / 2, \cdot}^{0}\right)_{j}=0, \\
& \left(L f_{i+1 / 2, .}^{1}\right)_{j}=-\frac{f_{i}^{0}-f_{i+1 / 2, j}^{0}}{h / 2} \cos \theta_{j}, \quad \text { if } \cos \theta_{j}>0, \\
& \left(L f_{i+1 / 2, .}^{1}\right)_{j}=\frac{f_{i+1}^{0}-f_{i+1 / 2, j}^{0}}{h / 2} \cos \theta_{j}, \quad \text { if } \cos \theta_{j}<0 .
\end{aligned}
$$

We first deduce from (4.19) that $f_{i+1 / 2, j}^{0}$ does not depend on $j$; we simply denote it by $f_{i+1 / 2}^{0}$. To compute

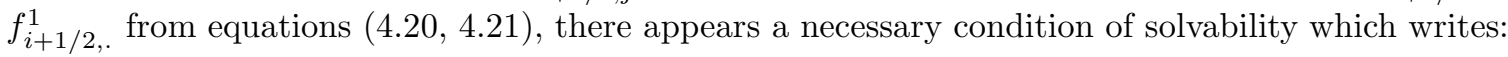

$$
-\left(\sum_{j, \cos \theta_{j}>0} \cos \theta_{j}\right) \frac{f_{i}^{0}-f_{i+1 / 2}^{0}}{\frac{h}{2}}+\left(\sum_{j, \cos \theta_{j}<0} \cos \theta_{j}\right) \frac{f_{i+1}^{0}-f_{i+1 / 2}^{0}}{\frac{h}{2}}=0
$$

i.e. $f_{i}^{0}-f_{i+1 / 2}^{0}+f_{i+1}^{0}-f_{i+1 / 2}^{0}=0$, on account of (3.6). We deduce that $f_{i+1 / 2}^{0}$ is the mean value of $f^{0}$ at points $x_{i}$ and $x_{i+1}$, i.e. if we have

$$
f_{i+1 / 2, j}^{0}=f_{i+1 / 2}^{0}=\frac{f_{i}^{0}+f_{i+1}^{0}}{2}
$$

Injecting this expression in $(4.20,4.21)$, we get, for all $j$ such that $\cos \theta_{j} \neq 0$ :

$$
\left(L f_{i+1 / 2, .}^{1}\right)_{j}=\frac{f_{i+1}^{0}-f_{i}^{0}}{h} \cos \theta_{j}
$$

which gives:

$$
f_{i+1 / 2, j}^{1}=Y_{j} \frac{f_{i+1}^{0}-f_{i}^{0}}{h}
$$

We now turn back to equations $(4.17,4.18)$. As $\left(D^{1 / 2} f^{0}\right)_{i, j}$ is independent of $j$, the solvability condition of equation (4.17) is satisfied. On account of expression (4.22), we have $\left(D^{1 / 2} f^{1}\right)_{i, j}=Y_{j}\left(\Delta_{h} f^{0}\right)_{i}$, so that the solvability condition of equation (4.18) simply writes:

$$
\partial_{t} f_{i}^{0}-\nu\left(\Delta_{h} f^{0}\right)_{i}=0
$$

which is exactly the discrete diffusion equation we expected, with the good coefficient of diffusion $\nu$ : this scheme seems to be the best one, which has been confirmed by numerical tests (see Figs. 5 and 6).

\subsection{A finite volume interpretation of the new scheme}

This new scheme can be interpreted as a generalization of the $P^{1}$ discontinuous finite elements scheme proposed by G. Samba (see [45]) for a stationary kinetic equation of Boltzmann-Lorentz type (see also [30,39]). But it can also be interpreted in terms of a classical finite volume scheme expressed on a refined mesh of size $h / 2$. We now detail this interpretation.

The aim is to explain the computation $(4.14,4.15)$ of the numerical flux. We first set $i^{\prime}=i-1 / 2$ and introduce the new discretization points $x_{i^{\prime}}=i^{\prime} h$ and $x_{i^{\prime}+k / 4}=\left(i^{\prime}+k / 4\right) h$, for $k \in \mathbb{Z}$. We set:

$$
f_{i+1 / 2, j}=\frac{1}{2}\left[f_{i^{\prime}+3 / 4, j}+f_{i^{\prime}+5 / 4, j}\right]
$$


where $f_{i^{\prime}+3 / 4, j}$ and $f_{i^{\prime}+5 / 4, j}$ solve the following equations:

$$
\begin{aligned}
& \varepsilon \partial_{t} f_{i^{\prime}+3 / 4, j}+\cos \theta_{j} \frac{F_{i^{\prime}+1, j}-F_{i^{\prime}+1 / 2, j}}{h / 2}=\frac{1}{\varepsilon}\left(L f_{i^{\prime}+3 / 4, .}\right)_{j}, \\
& \varepsilon \partial_{t} f_{i^{\prime}+5 / 4, j}+\cos \theta_{j} \frac{F_{i^{\prime}+3 / 2, j}-F_{i^{\prime}+1, j}}{h / 2}=\frac{1}{\varepsilon}\left(L f_{i^{\prime}+5 / 4, .}\right)_{j},
\end{aligned}
$$

with:

$$
\begin{aligned}
F_{i^{\prime}+1 / 2, j} & =\frac{1}{2}\left[f_{i^{\prime}+1 / 4, j}+f_{i^{\prime}+3 / 4, j}\right], \\
F_{i^{\prime}+1, j} & = \begin{cases}f_{i^{\prime}+3 / 4, j} & \text { if } \cos \theta_{j}>0, \\
f_{i^{\prime}+5 / 4, j} & \text { if } \cos \theta_{j}<0 .\end{cases}
\end{aligned}
$$

In the same way, we also naturally have:

$$
\varepsilon \partial_{t} f_{i^{\prime}+1 / 4, j}+\cos \theta_{j} \frac{F_{i^{\prime}+1 / 2, j}-F_{i^{\prime}, j}}{h / 2}=\frac{1}{\varepsilon}\left(L f_{i^{\prime}+1 / 4, .}\right) j .
$$

Now equations (4.23) and (4.24) give by addition:

$$
\varepsilon \partial_{t} f_{i+1 / 2, j}+\cos \theta_{j} \frac{F_{i^{\prime}+3 / 2, j}-F_{i^{\prime}+1 / 2, j}}{h}=\frac{1}{\varepsilon}\left(L f_{i+1 / 2, .}\right)_{j},
$$

or equivalently:

$$
\varepsilon \partial_{t} f_{i+1 / 2, j}+\cos \theta_{j} \frac{F_{i+1, j}-F_{i, j}}{h}=\frac{1}{\varepsilon}\left(L f_{i+1 / 2, .}\right)_{j} .
$$

We here recover the equation of evolution (4.1) expressed at the new spatial grid point $x_{i+1 / 2}$. It remains to find an equation for the flux $F_{i, j}$ at the interface of the refined mesh. This one is obtained by summing equations (4.23-4.25); we get:

$$
\varepsilon \partial_{t} F_{i, j}+\cos \theta_{j} \frac{F_{i^{\prime}+1, j}-F_{i^{\prime}, j}}{h}=\frac{1}{\varepsilon}\left(L F_{i, .}\right)_{j} .
$$

A simple computation first shows that:

$$
\frac{F_{i^{\prime}+1, j}-F_{i^{\prime}, j}}{h}= \begin{cases}\frac{2}{h}\left[F_{i, j}-f_{i-1 / 2, j}\right] & \text { for } \cos \theta_{j}>0, \\ \frac{2}{h}\left[f_{i+1 / 2, j}-F_{i, j}\right] & \text { for } \cos \theta_{j}<0 .\end{cases}
$$

Now, neglecting the lowest order term (with respect to $\varepsilon$ ) in (4.26), we get:

$$
\begin{array}{ll}
\frac{2 \cos \theta_{j}}{h}\left[F_{i, j}-f_{i-1 / 2, j}\right]=\frac{1}{\varepsilon}\left(L F_{i, .}\right)_{j}, & \text { if } \cos \theta_{j}>0, \\
\frac{2 \cos \theta_{j}}{h}\left[f_{i+1 / 2, j}-F_{i, j}\right]=\frac{1}{\varepsilon}\left(L F_{i, .}\right)_{j}, & \text { if } \cos \theta_{j}<0,
\end{array}
$$


or equivalently, for $i^{\prime}=i+1 / 2$ :

$$
\begin{aligned}
f_{i^{\prime}, j} & =F_{i^{\prime}+1 / 2, j}-\frac{h}{2 \varepsilon \cos \theta_{j}}\left(L F_{i^{\prime}+1 / 2, .}\right)_{j}, \quad \text { if } \cos \theta_{j}>0, \\
f_{i^{\prime}+1, j} & =F_{i^{\prime}+1 / 2, j}+\frac{h}{2 \varepsilon \cos \theta_{j}}\left(L F_{i^{\prime}+1 / 2, .}\right)_{j}, \quad \text { if } \cos \theta_{j}<0,
\end{aligned}
$$

i.e. we exactly recover formulae $(4.14,4.15)$ at the new grid point $x_{i^{\prime}}$.

\section{The time DiscretizAtion}

We now present the discretization in time. We first remark that it is of no use to apply a splitting in time method. In fact, for the first time step, the collision part

$$
\varepsilon \partial_{t} f=\frac{1}{\varepsilon} \mathcal{L} f
$$

would project on the constants with respect to the velocity angle $\theta$, and the transport part

$$
\varepsilon \partial_{t} f+\cos \theta \partial_{x} f=0
$$

would project on the constant with respect to the position variable $x$. Thus, in two time steps we will find a constant function (and not the good diffusion equation). The fact that splitting also fails in the diffusion limit is not surprising and we also refer to the Section A.2 where a similar problem occurs for the directional splitting in the multi-dimensional case.

On the other hand, when using an explicit scheme, we get time step restrictions. Due to the diffusive scaling of the equation (1.1), the time step stability condition associated with the transport term is of the form

$$
\Delta t \leq \varepsilon \Delta x
$$

since the velocities are of modulus smaller than 1, whereas the time step condition for the collision part reads:

$$
\Delta t \leq \tau \varepsilon^{2}(\Delta v)^{2}
$$

where $\tau$ is the collision time (equal 1 in this paper). Since the aim of the studied schemes is to be used for arbitrary small values of $\varepsilon$, the cost of such explicit schemes will be prohibitive especially due to the collision part.

Therefore, we shall always use implicit schemes for the collision part. For the transport part, we can use either fully explicit scheme (with the restriction given by (5.1)), semi-implicit scheme (for example using the $\varepsilon$-scheme described in Sect. 4.3) or a fully implicit scheme. More precisely, in the semi-implicit scheme, we implicit the collision and $(1-\varepsilon) D^{c}$, the first part of the transport, and treat explicitly the upwind transport part $\varepsilon D^{u}$. This leads us to a stability condition of the form $\Delta t \leq \Delta x$.

From numerical point of view, the semi and fully implicit scheme requires to invert $N_{x} \times N_{\theta}$ matrices where $N_{x}$ (respectively $N_{\theta}$ ) is the number of point in the discretization with respect to $x$ (respectively $v$ ).

\section{Numerical RESUlts}

The numerical tests are devoted to verify that the proposed discretization gives the right diffusion coefficient. We consider an initial data equal to a Dirac measure in $x$ and uniform in $\theta$ or concentrated in $\theta$ (but this latter choice does not change the solutions when $\varepsilon \rightarrow 0)$ :

$$
f(x, \theta, t=0)=\delta_{x=0} \text { or } f(x, \theta, t=0)=\delta_{x=0} \delta_{\theta=0}
$$




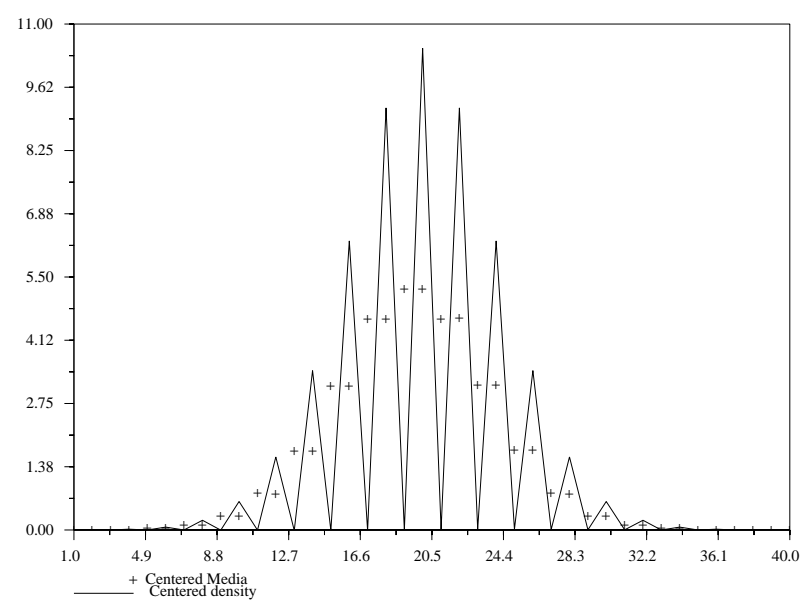

Figure 3. Centered scheme.

For such initial data, the solution $f^{\varepsilon}$ of problem (1.1) behaves when $\varepsilon \rightarrow 0$ as the solution $f^{0}$ of the heat equation, which is given by:

$$
f^{0}(x, t)=\frac{1}{\sqrt{4 \pi \nu t}} \exp \left(-\frac{x^{2}}{4 \nu t}\right) .
$$

In particular, the second moment of the solution is close to that of a Gaussian

$$
\int f^{\varepsilon}(t, x, \theta) x^{2} \mathrm{~d} x \mathrm{~d} \theta \propto 2 \nu t
$$

In other words, the evolution of the second moment of $f^{\varepsilon}$ becomes linear in time as $\varepsilon \rightarrow 0$ with a slope related to the diffusion coefficient of the limiting diffusive equation.

In the results presented here, we use the four velocities case presented in Section 3.2 for a given angle $\theta_{0}$. Here, and also in the following figures, $\varepsilon=0.001$, the numerical scheme is implicit in time, $\Delta x=40, \Delta \theta=4$, $\Delta t=0.01$ and time equal to 1 (i.e. 100 iterations).

In Figure 3, we plot the particle density $n(x, t)$,

$$
n(x, t)=\int f(x, \theta, t) \mathrm{d} \theta
$$

for the centered scheme. In particular, there are underlined the numerical oscillations due to the non-coupling between the odd and even meshes. Finally, the dotted line is a post-treated curve giving the mean values of $n(x, t)$ between two successive meshes.

The density $n(x, t)$ for the $\varepsilon$-scheme is plotted in Figure 4. We remark that the numerical oscillations in Figure 3 are smoothened by the effect of the upwind scheme. The difference between the diffusion coefficient in the $\varepsilon$-scheme and the centered scheme is not remarkable due to the fact that $h=\Delta x$ is small. The continuous line corresponds to the "optimal" angle computed for the nonuniform grid with four points, while the dotted line corresponds to the density $n(x, t)$ computed for the angle $\theta_{0}=\pi / 6$.

In Figure 5, we plot the density $n(x, t)$ for the modified Jin-Levermore scheme. The numerical oscillations have disappeared, and the diffusion coefficient is exactly the same that in the centered scheme. The continuous line corresponds to the "optimal" angle computed for the nonuniform grid with four points, while the dotted line corresponds to an angle equal to $\pi / 6$. 


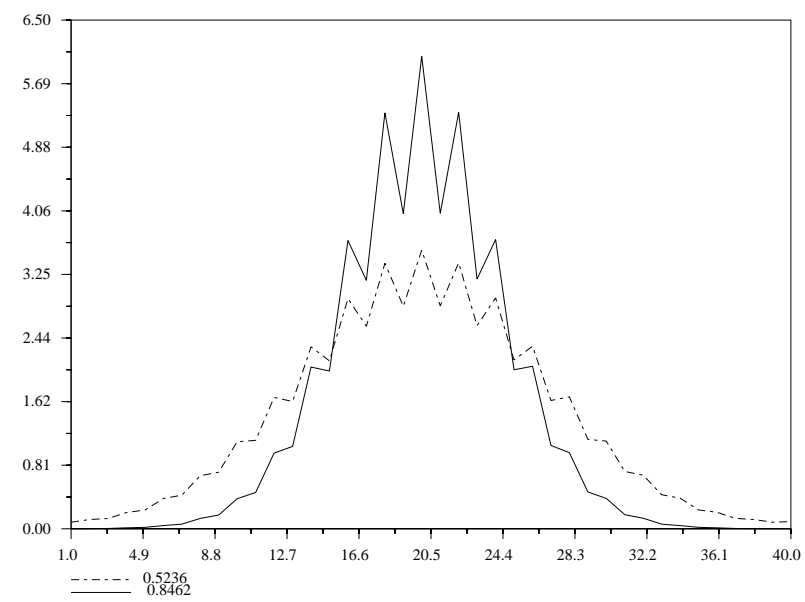

FiguRE 4. $\varepsilon$-scheme.

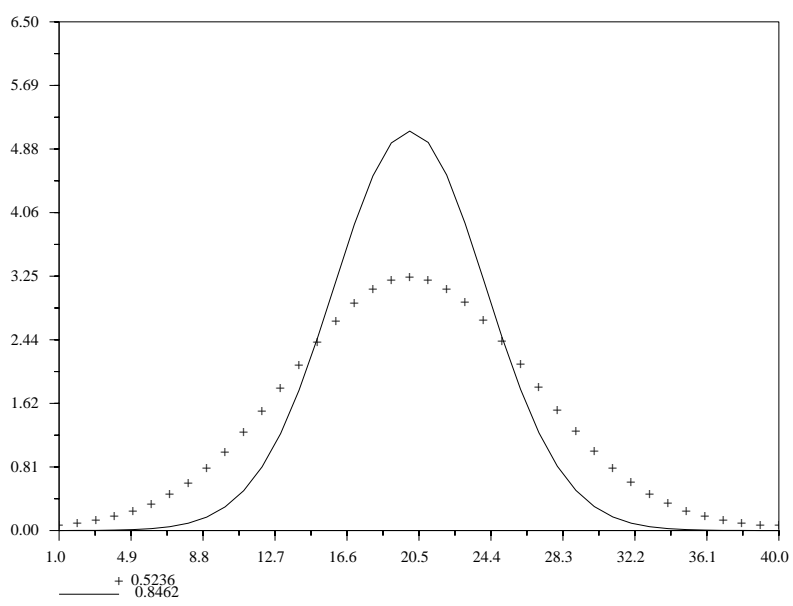

Figure 5. Modified Jin-Levermore scheme.

In Figure 6, we compare the two densities in the case of the centered scheme (the means value curve) and in the case of the modified Jin-Levermore scheme. We note that the Dirac mass has diffused in the same way, as it was attended from the previous analysis.

In Figure 7, we plot the curves of the diffusion coefficient $\nu(\theta)$ computed from the second moment, when $\theta$ is varying. We compare the result found for the exact solution of the heat equation with the one obtained by means of the modified Jin-Levermore scheme. Both the curves intersect the value $1 / 2$ for the optimal value of the angle $\theta_{0}=0.864$.

In Figure 8 we plot the density $n(x, t)$ for four different values of $\varepsilon$ at time 1 . For small values of $\varepsilon$ ( 0.01 and $0.00001)$ the curves are indistinguishable. For "large" values of $\varepsilon$ (larger than 1), the solution is very close to the initial data. For intermediate values $(\varepsilon=0.5)$, one observes the splitting of the initial delta measure into two picks: one moving to the right the other to the left, as expected in such kinetic model.

Finally, in Figure 9 we plot the diffusion coefficient curves in function of $\varepsilon=0.01, \ldots, 0.1$ for three different values of the discretization angle $\theta_{0}=\pi / 6, \pi / 3,0.864$. We note that for $\theta_{0}=0.864$ (which is the angle computed by Maple for the non-uniform four points discretization) the diffusion coefficient $\nu$ is close to $1 / 2$ when $\varepsilon=0.01$. 


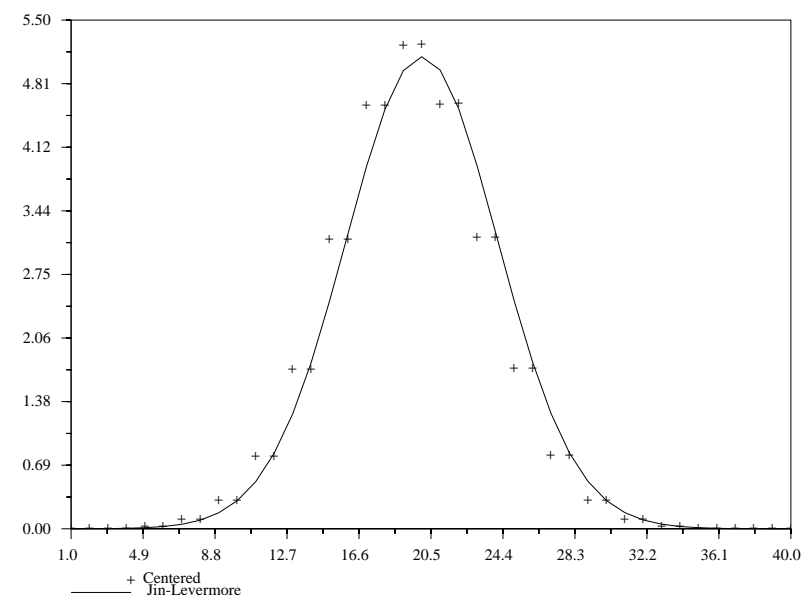

Figure 6. Modified Jin Levermore-centered scheme.

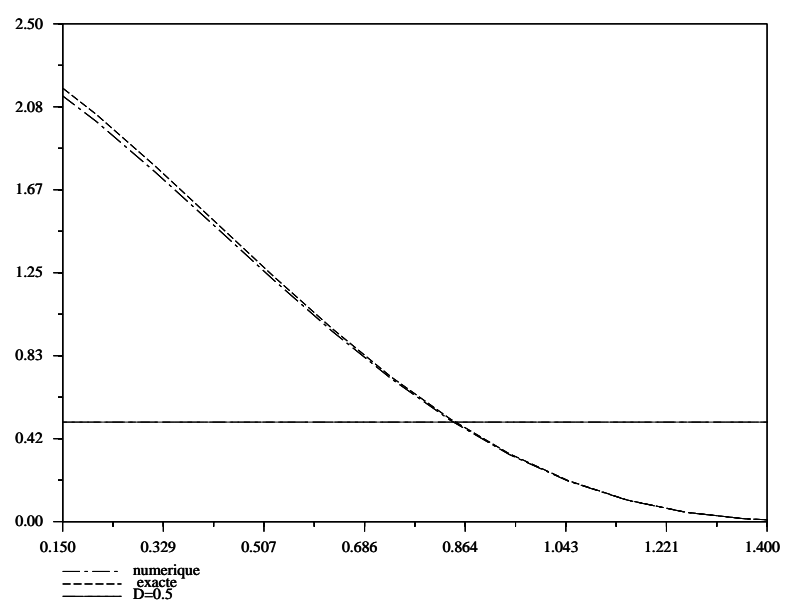

FIGURE 7. Diffusion coefficient w.r.t. $\theta$.

\section{Conclusions}

We first remark that in the $\varepsilon$ scheme proposed in Section 4.3, it is possible to replace the upwind scheme by the modified Jin-Levermore scheme of Section 4.4, that has all the required properties for $\varepsilon \leq h$. On the other hand, if $\varepsilon \geq h$, then the upwind scheme seems sufficient. Thus, the ultimate choice for the discretization in space is, for example,

$$
D=\max (0,1-\varepsilon / h) D^{1 / 2}+\min (1, \varepsilon / h) D^{u} .
$$

Let us emphasize that every method leading to the good diffusion equation (see for example [15,24-26]), requires the inversion of a huge (but sparse) matrix, of size $N_{x} \times N_{\theta}$ where $N_{x}$ is the number of discretization points in space and $N_{\theta}$ is the number of discretization points in velocity. In fact, one needs implicit schemes in order to avoid too restrictive time step condition in terms of $\varepsilon$, and a splitting of space and velocity is not possible. Indeed, for all these methods in order to compute the fluxes we must solve a stationary problem (see for example 


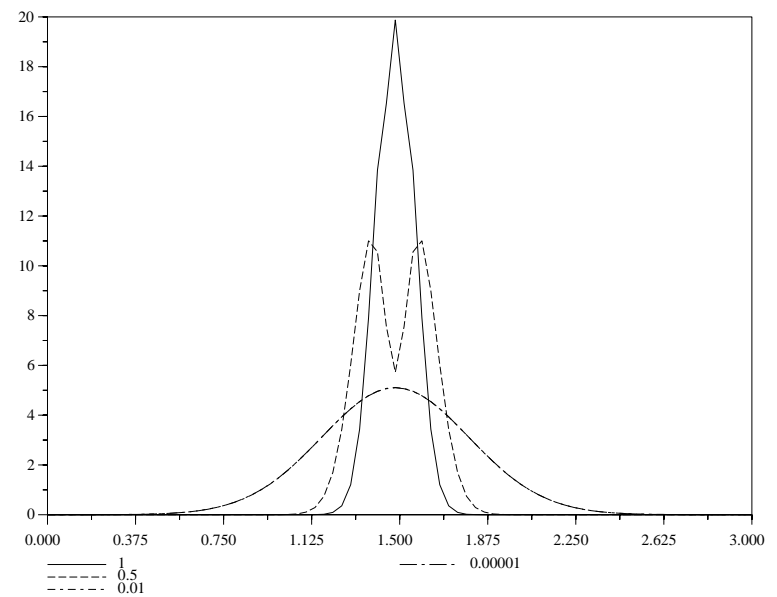

Figure 8. Density for three values of $\varepsilon$.

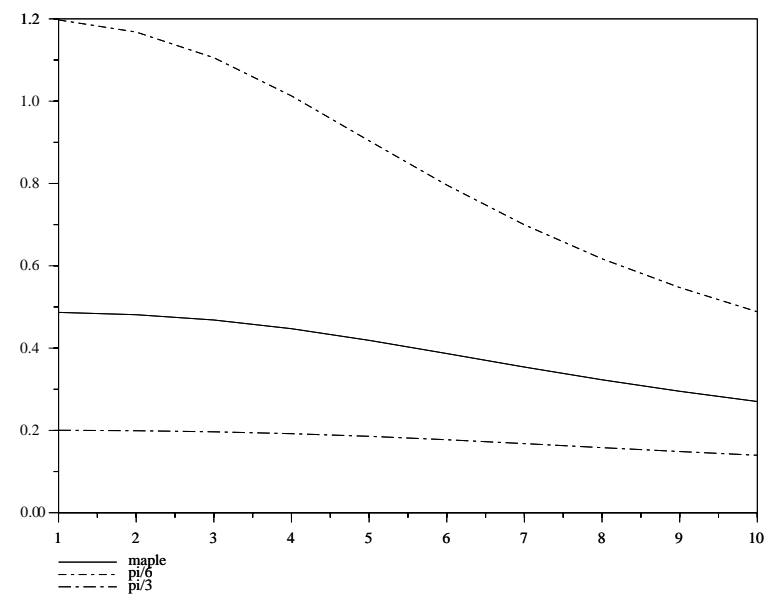

Figure 9. Diffusion coefficient $\nu$ versus $\varepsilon$.

equation (2.3)) of the type:

$$
v \cdot \nabla_{x} f^{0}=\mathcal{L}\left(f^{1}\right)
$$

Such a problem is necessarily multi-dimensional, i.e. one cannot split space directions $x$ and velocity variables $v$. We also remark that there is no non-trivial hydro-dynamical limit (see Sect. A.1). Moreover, the bi-dimensional case with respect to the space variable can be treated by a similar approach but the directional splitting does not give the good diffusion coefficient (see Sect. A.2). Nevertheless, the finite volume approach described in Section 4.5, could be a way to design a fully multi-dimensional asymptotic preserving scheme.

Some questions remain open:

- Take into account boundary condition (or equivalently bounded region in $x$ ): as in [13,22-24], the solution has a boundary layer as $\varepsilon \rightarrow 0$ which is treated using extrapolation length.

- Generalize to a non uniform grid in space and analyze the two dimensional case (in space) with unstructured meshes, or other geometries with for example spherical or axial symmetry. 
- Consider inelastic collisions or force fields that couples the kinetic energy level i.e. consider a distribution function depending of the kinetic energy.

- Numerical test with regions where transport dominates $(\varepsilon \gg 1)$ and other highly collisional $(\varepsilon \ll 1)$ are needed in order to validate the choice proposed in (7.1).

- Study the positiveness and other properties of the solution when using Hilbert expansion method.

\section{A. Appendix: SOME FinAl REMARKS}

\section{A.1. Hydro-dynamical limit}

Consider the Boltzmann kinetic equation at a hydrodynamic regime:

$$
\partial_{t} f+\cos \theta \partial_{x} f=\frac{1}{\varepsilon} \mathcal{L}(f) .
$$

Is it possible to make the same analysis as for the diffusion regime. We begin by the continuous level with $\mathcal{L}(f)=\partial_{\theta \theta}^{2} f$. Expanding $f$ in powers of $\varepsilon$, and identifying the equal orders, we get:

$$
\begin{aligned}
0 & =\mathcal{L}\left(f^{0}\right), \\
\partial_{t} f^{0}+\cos \theta \partial_{x} f^{0} & =\mathcal{L}\left(f^{1}\right), \\
\partial_{t} f^{1}+\cos \theta \partial_{x} f^{1} & =\mathcal{L}\left(f^{2}\right) .
\end{aligned}
$$

Then solving equation (A.1) gives that $f^{0}$ is independent on the $\theta$ variable: $f^{0}=f^{0}(x, t)$. The solvability condition for equation (A.2) reads:

$$
2 \pi \partial_{t} f^{0}+\int_{0}^{2 \pi} \cos \theta \partial_{x} f^{0} \mathrm{~d} \theta=0
$$

which implies $f^{0}$ independent on $t$, too. Thus, $f^{0}=f^{0}(x)$ and $f^{1}=f^{1}(x, \theta)=-\cos \theta \partial_{x} f^{0}$. Finally, the solvability condition for equation (A.3) is given by:

$$
\int_{0}^{2 \pi}-(\cos \theta)^{2} \partial_{x}^{2} x f^{0}=0
$$

which first gives $f^{0}=C^{\prime} x+C$ but, thanks to the behavior at infinity, we finally get $f^{0}=C$; we also deduce that $f^{1}=0$. Thus, it shows that the hydro-dynamical limit is trivial.

\section{A.2. Bi-dimensional case}

Let us consider a two dimensional case. We shall show that a directional splitting is not suitable since it leads to a wrong diffusion coefficient. We shall present the analysis on the simplest, centered case.

We denote by $i$ the index for the $x$ position variable, by $k$ the index for the $y$ position variable. For simplicity, we shall consider only the discretization with respect to the position variables $x$ and $y$, keeping the velocity $\theta$ and the time $t$ at a continuous level. Applying a centered scheme to the kinetic equation, leads to:

$$
\varepsilon \partial_{t} f_{i, k}+\cos \theta D^{1} f_{i, k}+\sin \theta D^{2} f_{i, k}=\frac{1}{\varepsilon} \mathcal{L} f_{i, k},
$$

where

$$
D^{1} f_{i, k}=\left(\frac{f_{i+1, k}-f_{i-1, k}}{2 \Delta x}\right)
$$


and

$$
D^{2} f_{i, k}=\left(\frac{f_{i, k+1}-f_{i, k-1}}{2 \Delta y}\right)
$$

Performing the Hilbert expansion method with

$$
f_{i, k}=f_{i, k}^{0}+\varepsilon f_{i, k}^{1}+\varepsilon^{2} f_{i, k}^{2}
$$

we get at the order $\varepsilon^{-1}$ :

$$
f_{i, k}=f_{i, k}^{0}=\rho_{i, k}, \quad \text { independent on } \theta
$$

Then at the order $\varepsilon^{0}$ we obtain:

$$
\cos \theta D^{1} f_{i, k}^{0}+\sin \theta D^{2} f_{i, k}^{0}=\mathcal{L} f_{i, k}^{1},
$$

which gives:

$$
f_{i, k}^{1}=-\cos \theta D^{1} f_{i, k}^{0}-\sin \theta D^{2} f_{i, k}^{0} .
$$

Finally, at the order $\varepsilon^{1}$, we have:

$$
\partial_{t} f_{i, k}^{0}+\cos \theta D^{1} f_{i, k}^{1}+\sin \theta D^{2} f_{i, k}^{1}=\mathcal{L} f_{i, k}^{2}
$$

and integrating with respect to $\theta$ and replacing $f^{1}$ by (A.5), this yields to the diffusion equation on a double mesh:

$$
\partial_{t} \rho_{i, k}-\frac{1}{2}\left(\frac{\rho_{i+2, k}+\rho_{i-2, k}-2 \rho_{i, k}}{(2 \Delta x)^{2}}\right)-\frac{1}{2}\left(\frac{\rho_{i, k+2}+\rho_{i, k-2}-2 \rho_{i, k}}{(2 \Delta y)^{2}}\right)=0 .
$$

Still this discretization has the same problems of the one in the one-dimensional case: it does not couple the odd and even grid points of the mesh.

We remark also that the same analysis of the modified Jin-Levermore scheme can be performed when using a rectangular structured mesh but it is much more complicated with a general mesh, see $[18,19,45]$ for works in this direction. Finally, it seems that a directional splitting (alternative directions) for the modified Jin-Levermore scheme leads to a wrong diffusion coefficient. Indeed, (A.4) can be splitted in two parts:

$$
\varepsilon \partial_{t} f_{i, k}=\left(-\cos \theta D^{1} f_{i, k}+\frac{1}{2 \varepsilon} \mathcal{L} f_{i, k}\right)+\left(-\sin \theta D^{2} f_{i, k}+\frac{1}{2 \varepsilon} \mathcal{L} f_{i, k}\right) .
$$

The first bracket corresponds to the derivative with respect to $x$ and half of the collision, the second bracket corresponds to the derivative with respect to $y$ and half of the collision. Then, using a splitting, one solves the first part which gives when $\varepsilon$ tends to zero, the diffusion equation in $x$ with coefficient $\nu_{x}=1 / 4$, instead of $1 / 2$. Thus, in order to recover the right diffusion coefficient one may not use a directional splitting.

\section{REFERENCES}

[1] M.L. Adams, Subcell balance methods for radiative transfer on arbitrary grids. Transport Theory Statist. Phys. 27 (1997) 385-431.

[2] R. Botchorishvili, B. Perthame and A. Vasseur, Equilibrium schemes for scalar conservation laws with stiff sources. Inria report RR-3891 (2000), http://www.inria.fr/RRRT/RR-3891.html 
[3] C. Buet, S. Cordier and B. Lucquin-Desreux, The grazing collision limit for the Boltzmann-Lorentz model. Asymptot. Anal. 25 (2001) 93-107.

[4] R.E. Caflisch, S. Jin and G. Russo, Uniformly accurate schemes for hyperbolic systems with relaxation. SIAM J. Numer. Anal. 34 (1997) 246-281.

[5] G.Q. Chen, C.D. Levermore and T.P. Liu, Hyperbolic conservation laws with stiff relaxation terms and entropy. Comm. Pure Appl. Math. 47 (1994) 187-830.

[6] S. Cordier, B. Lucquin-Desreux and A. Sabry, Numerical approximation of the Vlasov-Fokker-Planck-Lorentz model. ESAIM: Procced. CEMRACS 1999 (2001), http://www.emath.fr/Maths/Proc/Vol.10

[7] P. Degond and B. Lucquin-Desreux, The Fokker-Planck asymptotics of the Boltzmann collision operator in the Coulomb case. Math. Models Methods Appl. Sci. 2 (1992) 167-182.

[8] P. Degond and B. Lucquin-Desreux, The asymptotics of collision operators for two species of particles of disparate masses. Math. Models Methods Appl. Sci. 6 (1996) 405-436.

[9] L. Desvillettes, On asymptotics of the Boltzmann equation when the collisions become grazing. Transport Theory Statist. Phys. 21 (1992) 259-276.

[10] J. Glimm, G. Marshall and B.J. Plohr, A generalized Riemann problem for quasi one dimensional gas flows. Adv. in Appl. Math. 5 (1984) 1-30.

[11] E. Godlewski and P.A. Raviart, Numerical approximations of hyperbolic systems of conservation laws. Springer-Verlag, New York, Appl. Math. Sci. 118 (1996).

[12] S. Goldstein, On diffusion by discontinuous movements, and on the telegraph equation. Quart. J. Mech. Appl. Math. 4 (1951) $129-156$.

[13] F. Golse, S. Jin and C.D. Levermore, The convergence of numerical transfer schemes in diffusive regimes I: discrete-ordinate method. SIAM J. Numer. Anal. 36 (1999) 1333-1369.

[14] L. Gosse, A priori error estimate for a well-balanced scheme designed for inhomogeneous scalar conservation laws. C. R. Acad. Sci. Paris Sér. I Math. 327 (1998) 467-472.

[15] L. Gosse, A well-balanced scheme using non-conservative products designed for hyperbolic systems of conservation laws with source terms. Math. Models Methods Appl. Sci. 11 (2001) 339-365.

[16] L. Gosse and A.Y. Leroux, A well-balanced scheme designed for inhomogeneous scalar conservation laws. C. R. Acad. Sci. Paris Sér. I Math. I323 (1996) 543-546.

[17] J.M. Greenberg and A.Y. Leroux, A well balanced scheme for the numerical processing of source terms in hyperbolic equations. SIAM J. Numer. Anal. 33 (1996) 1-16.

[18] F. Hermeline, A finite volume method for the approximation of diffusion operators on distorted meshes. J. Comput. Phys 160 (2000) 481-499.

[19] F. Hermeline, Two coupled particle-finite volume methods using Delaunay-Voronoï meshes for the approximation of VlasovPoisson and Vlasov-Maxwell equations. J. Comput. Phys 106 (1993).

[20] S. Jin, Efficient asymptotic-preserving (AP) schemes for some multiscale kinetic equations. SIAM J. Sci. Comput. 21 (1999) 441-454.

[21] S. Jin, Numerical integrations of systems of conservation laws of mixed type. SIAM J. Appl. Math. 55 (1995) $1536-1551$.

[22] S. Jin and C.D. Levermore, The discrete-ordinate method in diffusive regimes. Transport Theory Statist. Phys. 20 (1991) 413-439.

[23] S. Jin and C.D. Levermore, Fully-discrete numerical transfer in diffusive regimes. Transport Theory Statist. Phys. 22 (1993) 739-791.

[24] S. Jin and C.D. Levermore, Numerical schemes for hyperbolic conservation laws with stiff relaxation terms. J. Comput. Phys. 126 (1996) 449-467.

[25] S. Jin and L. Pareschi, Discretization of the multiscale semiconductor Boltzmann equation by diffusive relaxation schemes. $J$. Comput. Phys. 161 (2000) 312-330.

[26] S. Jin, L. Pareschi and G. Toscani, Diffusive relaxation schemes for multiscale discrete-velocity kinetic equations. SIAM J. Numer. Anal. 35 (1998) 2405-2439.

[27] S. Jin, L. Pareschi and G. Toscani, Uniformly accurate diffusive relaxation schemes for multiscale transport equations. SIAM J. Numer. Anal. (2000).

[28] S. Jin and Z. Xin, The relaxation schemes for systems of conservation laws in arbitrary space dimensions. Comm. Pure Appl. Math. XLVIII (1995) 235-276.

[29] A. Klar, An asymptotic-induced scheme for non stationary transport equations in the diffusive limit. SIAM J. Numer. Anal 35 (1998) 1073-1094.

[30] E.W. Larsen, The asymptotic diffusion limit of discretized transport problems. Nuclear Sci. Eng. 112 (1992) $336-346$.

[31] E.W. Larsen and J.E. Morel, Asymptotic solutions of numerical transport problems in optically thick, diffusive regimes. II. $J$. Comput. Phys. 83 (1989) 212-236.

[32] E.W. Larsen, J.E. Morel and W.F. Miller Jr., Asymptotic solutions of numerical transport problems in optically thick, diffusive regimes. J. Comput. Phys. 69 (1987) 283-324. 
[33] R.J. LeVeque, Balancing source terms and flux gradients in high-resolution Godunov methods: the quasi-steady wavepropagation algorithm. J. Comput. Phys. 146 (1998) 346-365.

[34] P.L. Lions, B. Perthame and P.E. Souganidis, Existence of entropy solutions for the hyperbolic systems of isentropic gas dynamics in Eulerian and Lagrangian coordinates. Comm. Pure Appl. Math. 49 (1996) 599-638.

[35] B. Lucquin-Desreux, Diffusion of electrons by multicharged ions. Math. Models Methods Appl. Sci. 10 (2000) 409-440.

[36] B. Lucquin-Desreux and S. Mancini, A finite element approximation of grazing collisions (submitted).

[37] P.A. Markowich, C. Ringhoffer and C. Schmeiser, Semiconductor equations. Springer-Verlag (1994).

[38] W.F. Miller Jr. and T. Noh, Finite differences versus finite elements in slab geometry, even-parity transport theory. Transport Theory Statist. Phys. 22 (1993) 247-270.

[39] J.E. Morel, T.A. Wareing and K. Smith, A linear-discontinuous spatial differencing scheme for $S_{n}$ radiative transfer calculations. J. Comput. Phys. 128 (1996) 445-462.

[40] G. Naldi and L. Pareschi, Numerical schemes for kinetic equations in diffusive regimes. Appl. Math. Lett. 11 (1998) $29-55$.

[41] L. Pareschi, Central differencing based numerical schemes for hyperbolic conservation laws with relaxation terms. J. Num. Anal. (to appear).

[42] B. Perthame, An introduction to kinetic schemes for gas dynamics. An introduction to recent developments in theory and numerics for conservation laws. L.N. in Computational Sc. and Eng., 5, D. Kroner, M. Ohlberger and C. Rohde Eds., Springer (1998).

[43] K.H. Prendergast and K. Xu, Numerical hydrodynamics for gas-kinetic theory. J. Comput. Phys. 109 (1993) 53-66.

[44] K.H. Prendergast and K. Xu, Numerical Navier-Stokes solutions from gas kinetic theory. J. Comput. Phys. 114 (1994) 9-17.

[45] G. Samba, Limite asymptotique d'un schéma d'éléments finis linéaires discontinus lumpés en régime diffusion. Rapport CEA (to appear).

[46] G.I. Taylor, Diffusion by continuous movements. Proc. London Math. Soc. 20 (1921) 196-212.

[47] B. Vanleer, On the relation between the upwind differencing schemes of Engquist-Osher, Godunov and Roe. SIAM J. Sci. Stat. Comp. 5 (1984) 1-20.

To access this journal online: www.edpsciences.org 